\title{
REVALORIZAR LA PRODUCCIÓN ACADÉMICA. UNA PREMISA FUNDANTE CONTRA LA PRECARIZACIÓN DEL TRABAJO EN EDUCACIÓN SUPERIOR
}

\author{
Gustavo Brufman (Universidad Nacional de Rosario** \\ gbrufman@gmail.com
}

Recibido: 7/08/2012 Aceptado: 10/12/2012

\section{Resumen}

El presente artículo se presenta como una suerte de ensayo de carácter iniciático en aras de extender el debate teórico-conceptual a partir de una serie de intercambios y reflexiones sobre nuestra práctica docente y su inscripción laboral. Se trata de la necesidad de allanar el camino hacia el develamiento de claves para un proceso de progresiva desmercantilización del conocimiento académico/científico, realzando la especificidad de nuestra labor docente. La vigencia aún de la Ley №24.521 de Educación Superior (1995) que desreguló y flexibilizó los procesos de trabajo docentes en este nivel educativo, nos conmina a la búsqueda de nuevas estrategias colectivas que ubiquen en el centro de nuestra atención el sentido mismo que ha adquirido en las universidades nacionales la profesión docente y su valorización como actividad productiva. El objetivo es ampliar el análisis abriendo categorías que favorezcan los intercambios en el sentido propuesto en momentos que nacionalmente se discute en las Comisiones Paritarias del sector, un Convenio Laboral único y la institucionalización de un proyecto de Carrera Docente común a todas las universidades nacionales.

\section{Palabras clave}

Trabajo - Precarización - Profesión Académica - Regulación - Cultura Académica.

\footnotetext{
Abstract

This article is presented as an introductory essay intended to expand the theoretical and conceptual debate arising from a series of discussions and ideas regarding our

* Licenciado en Ciencias de la Educación. Universidad Nacional de Rosario. Investigador del Consejo de Investigaciones de la Universidad Nacional de Rosario.
} 
teaching experience and its employment implications. This paper is also intended to remove the obstacles in order to reveal the keys of a process of progressive decommercialization of academic/scientific knowledge, enhancing the specificity of our teaching job. The current Higher Education Act (Ley de Educación Superior) \# 24.521, from 1995, which deregulated and led to the flexibility of the teaching working processes at this education level, makes it necessary for us to find new collective strategies focused on finding the purpose of the teaching profession within the national universities, and recovering its value as a productive activity. The goal is to extend the analysis by including categories that encourage exchanges in the proposed direction. at a time when national collective bargaining negotiations are taking place in this field regarding the implementation of a unique Employment Agreement and the institutionalization of a Teaching Career project that is common to every national university.

\section{Key words}

Work - Precariousness - Academic Profession - Regulation-Academic Culture.

\section{Introducción}

Nos encontramos instalados en un contexto de disputa teórica desde el punto de vista conceptual y político en el campo de la regulación del trabajo académico, tomando a esta como "los procedimientos que una institución o conjunto de ellas establecen para el ingreso, la permanencia y la promoción de la carrera académica" (Zuñiga Hernández, O. y otros, 2011: p.107). Procedimientos desplegados en el marco de una clara tendencia según la cual, en la facticidad de los hechos, se considera a la propia tarea de la enseñanza en la Educación Superior -según el autor mencionado- como una suerte de mera actividad narrativa (de comentarios de textos y autores) mientras la investigación y el conocimiento codificado (1) se imponen como columna vertebral a la cual se alinean actividades subsidiarias del trabajo académico.

De allí en adelante, el criterio extendido que domina la cultura académica de nuestras universidades -como hegemonía socialmente construida- es el que equipara la profesionalización de la investigación a la noción de profesionalización académica. Siendo que en rigor, esta última, refiere a un territorio mucho más vasto en el que hoy además, se inscribe la degradación -en términos valorativos- de la tarea estrictamente docente. $Y$ a nuestro saber y entender, por cuestiones ajenas no necesariamente propias del espacio universitario, como por ejemplo regímenes impuestos por la lógica del mercado del conocimiento producido a bajo costo, condicionando las agendas de investigación de docentes volcados mayormente a esta tarea por el financiamiento de grandes empresas.

Partimos de la idea de que la profesionalización de la enseñanza es un marco general que contiene a la profesionalización de la investigación, pero no es sustitutiva. Esto nos exige analizar la diversificación del trabajo académico 
y lo que podríamos concebir como sus "distorsiones" conceptuales a partir de las políticas instrumentadas en la educación superior. (Boyer, E.;1997) (2).

Preocupa, como variable significativa, la singular dinámica que adquiere en la presente coyuntura de las universidades latinoamericanas -argentinas en particular- y sus organizaciones sindicales a la hora de analizar aquellas regulaciones laborales, la dialéctica que se instala entre lo general y común de las políticas en educación superior adoptadas regionalmente y lo particular de los sujetos involucrados en las discusiones según sea su anclaje social, territorial, laboral concreto, al tener que discutir el proceso de valorización del trabajo docente..

Para pensar "lo académico" (y por ende directa o indirectamente, a "los académicos") resultan interesantes las producciones que sugieren identificaciones laborales singulares y específicas en la perspectiva que entiende al Proceso de Trabajo (PT) -para nuestro caso, docente- desde las aproximaciones teóricas propias de la Actividad Situada (AS) y del Conocimiento Socialmente Distribuido (CSD) (Lozares, C. y otros, 2004: p. 74) (3). Como así también, las que reafirman de alguna manera en esa misma línea de análisis, que:

...las identidades, representaciones y prácticas (IRyP) del mundo del trabajo no pueden ser comprendidas como puro discurso, sin prestar atención a la relación de este con las normas y reglas sociales. Señalando que las IRyP se desarrollan en el marco estructural y simbólico de las normas en vigencia, cualquiera sea el grado de creencia respecto de ellas, a las cuales producen, reproducen y transforman. (La Serna, C., 2010: p. 21).

El desafío presente pasaría entonces por encontrar un marco regulatorio que desde el punto de vista laboral, nos abarque, nos proteja y nos contenga con posibilidades en lo profesional de calificar y promocionar a través de un proceso de formación continua. Desafío que deberá atender el conjunto de tensiones que instalan las formas de producción a que nos remite la reestructuración del modelo universitario hegemónico por un lado. Particularmente en lo que refiere al nosotros trabajadores/as docentes universitarios como productores/as inmateriales que generamos conocimientos y producimos fuerza de trabajo. Y por el otro, a las continuidades -pese a los avances que podamos observar desde 2003 a la fecha- que se registran en los sistemas de Educación Superior (ES) de la región, en la perspectiva mercantilizadora del conocimiento sostenido por docentes altamente flexibilizados. Marco que nos proporcione previsibilidad, nos garantice estabilidad y acote la arbitrariedad a la que somos sometidos la inmensa mayoría de los universitarios, degradando (desde el modelo de universidad comercial (4) impuesto) una tarea (la estrictamente docente) en detrimento de otra (la investigación) impactando en las trayectorias académicas y el quehacer cotidiano en los espacios universitarios (Monfredini, I., 2011). 
Situación esta, que termina fracturando y segmentando concepción, gestión y ejecución de la educación tras un discurso de corte academicista/profesionalista que oculta más que lo que devela; acotando y reduciendo la dimensión intelectual de nuestra producción, en todas las áreas, funciones y tareas que configuran el proceso de trabajo universitario como dimensión compleja a estudiar en profundidad. Regulación que se instala mientras se reproduce (e incluso en ocasiones se profundiza) la precariedad laboral.

\section{Algunas observaciones necesarias}

Los trabajos que se presentaron en el IX Seminario Internacional de la RED ESTRADO (5) realizado en Chile los días 18, 19 y 20 de Julio de 2012 han girado fundamentalmente en torno a la precarización del trabajador docente universitario. Las tendencias expresadas en las investigaciones presentadas muestran que, de una forma u otra, el centro del debate vuelve a estar puesto en "el valor" del trabajo. Contexto que creemos, instala razones suficientes por las que resulta inexorable atender, dada la diversidad de intereses puestos en juego por el estado, la universidad y el mercado, la tensión entre Autonomía Universitaria y Evaluación, centrando la mirada en quién evalúa. Atendiendo a este contexto y a cómo se ponen en juego los valores académicos y los valores de mercado, es necesario indagar además en torno a los supuestos subyacentes en los criterios evaluadores sobre cada una de las dimensiones del proceso laboral.

En esta línea de razonamiento, resultan interesantes para encuadrar y contextualizar dichos supuestos, los avances en torno a la categoría de Pertinencia de la Educación Superior (PES) que formula Sebastián Gómez, cuando problematiza el binomio público-privado presupuesto en la categoría PES, al analizar los proyectos de Ley de Educación Superior de los distintos bloques parlamentarios que fueron elevados desde el año 2006 al año 2009. (Gómez, S.; 2009) (6).

El segundo punto sobre el que llamamos la atención, es acerca de la noción de precarización con que trabajamos en el desarrollo de la $\|^{\mathrm{a}}$ Parte del "Trayecto Curricular Sistemático de Formación Docente: Trabajo Docente en la Universidad. Una Investigación Participativa" (Ps. Deolidia Martinez y equipo. 2011) dictado en el marco del Programa Nacional de Formación Gratuita (Acta CIN-CONADU del 28/09/2011), en la Asociación Gremial de Docentes e Investigadores de la UNR (COAD), en el sentido de que:

...hay que abrir la categoría y dar entrada a TODAS las precarizaciones (no solo la carencia de derechos laborales): en los vínculos, la comunicación, la relación con los estudiantes, el trabajo colectivo en los procesos reales intervinculados (con los no-docentes) y en especial, hay que ver quién precariza, quién es el precarizador y qué política concreta lo expresa (ajuste, evaluación, 
valor de mercado, apertura a una demanda que no se tiene con qué atender, etc. (Martinez, D. 2011: p.3)

Un tercer punto, que de alguna manera se desprende de este y que nos remite a producciones anteriores, refiere a que, analizar cómo se trabaja en la universidad exige hoy incorporar al conjunto de todos los que directa o indirectamente forman parte del proceso de trabajo docente (estudiantes, administrativos, auxiliares, laboratoristas, etc.). Finalmente y por tal motivo, no consideramos un dato menor formulaciones tales como las que hiciera el Espacio Nacional de Estudiantes de Organizaciones de Base -ENEOB- (justamente, en tanto son parte misma del proceso laboral docente) tras multitudinarios FOROS de discusión, al elaborar sus 15 Puntos para una Nueva Ley de Educación Superior. (www.eneobargentina.com.ar). Allí especifican:

7. Las formas en que se lleva adelante la Evaluación deben bregar, en primer término, por la protección de la autonomía de la ES. Cualquier tipo de evaluación académica o institucional debe contemplar las siguientes condiciones:

a) La definición de criterios de evaluación que respeten la autonomía universitaria. Su ejecución debe ser realizada en forma independiente del poder ejecutivo, legislativo y judicial y de cualquier entidad privada.

b) Debe contemplar la participación de los estudiantes.

c) Debe contemplar la participación de otros sectores sociales como sindicatos, movimientos sociales y organizaciones de la sociedad civil.

d) Los resultados de los procesos de evaluación no pueden ser utilizados para definir la distribución de presupuesto, y no pueden acarrear la posibilidad de inhabilitación para expedir títulos.

Rechazamos la CONEAU y cualquier órgano de evaluación que use mecanismos extorsivos violatorios de la autonomía de la Educación.

8. La nueva LES debe abonar la conformación de mecanismos de autoevaluación democráticos y protectores de la autonomía de las Instituciones de Educación Superior, que cuenten con la participación de todos los claustros y donde exista representación de actores como los arriba mencionados. (p.2).

\section{La docencia universitaria y la profesionalización académica en universidades públicas con alta precarización}

El documento sobre Carrera Docente aprobado por la Federación Nacional de Docentes Universitarios (CONADU) en su último Congreso Extraordinario celebrado los días 21 y 22 de Junio 2012 en la ciudad de Formosa, subraya entre sus fundamentos para ser llevado a la discusión del Convenio Colectivo de Trabajo (CCT), que el Sistema Universitario Público Nacional que en 1918 contaba solamente con cuatro instituciones, se ha expandido notablemente y reúne hoy 47 universidades y 7 institutos universitarios. Sumando sus plantas, estaríamos hablando de un total de 140.000 docentes aproximadamente. 
Escenario político-académico que se produce con un cambio sustantivo en lo que hace a la vinculación de los propios docentes universitarios con su tarea y con la institución académica. Pero también, en la imagen que estos tienen de sí y de sus quehaceres. De manera creciente, especifica:

...los universitarios -que solían ser profesionales que dedicaban a la tarea académica sólo un tiempo menor de su actividad- procuran consolidar su situación laboral como docentes de tiempo completo en las instituciones, expectativa que se incrementa en la medida en que la actividad académica se profesionaliza y mejoran las condiciones salariales y las oportunidades de desarrollo personal que esta ocupación ofrece. (IEC-CONADU: p.6).

La contracara de esta tendencia que caracteriza de manera hegemónica a todo el sector, es que las designaciones interinas que debieran ser de carácter excepcional, se han consolidado como la forma normal de vinculación del docente a la universidad. El mismo documento afirma que en la actualidad los docentes no regulares u ordinarios representan más del $60 \%$ del sistema, llegando a superar el $70 \%$ en algunas universidades. Lo que se pensó originalmente como un recurso excepcional para cubrir vacantes de manera inmediata y provisoria que garantizara la continuidad de las actividades cuando se abrieran carreras o asignaturas, o frente al imprevisto por liberación de cargos, "se constituyó en la característica estructural de un modelo universitario de reducción de derechos por sometimiento y sujeción a un régimen de despido periódico". (IEC-CONADU, 2012: p.6). Uno de los debates que se mantuvieron en aquel Congreso y que se expresó a través de los docentes de la UNR y la UNRC, fue que atento a que la excepción y la irregularidad del sistema devino la característica con un alto nivel de "naturalización" para las gestiones del conjunto de las UU.NN., allí donde existe algún tipo de régimen de Carrera Docente, en general, lejos de tratarse de un mecanismo de promoción y profesionalización, apenas constituye un régimen de -pretendida- estabilidad laboral -según la discrecionalidad con que se aplique- reduciendo y tergiversando su sentido original y manteniendo además en paralelo aquella altísima proporción de docentes interinos.

El documento mencionado consigna a su vez que:

En sentido estricto, la cuestión de los interinos no forma parte del Régimen de Carrera Docente. Pero la implementación de la Carrera exige, al mismo tiempo, dar una respuesta a esta situación. No es admisible que la carrera docente sea opcional, o que se defina el ingreso de tal modo que haya en una institución dos clases de docentes con regímenes laborales distintos. La Carrera Docente es el modo de asegurar la estabilidad laboral, pero también es el instrumento que permite ordenar las plantas, proyectar las carreras académicas individuales y planificar el desarrollo institucional de acuerdo con objetivos definidos. El mantenimiento de un sector de la planta docente bajo el viejo régimen de la periodicidad, o, peor aún, bajo el interinato, reserva un 
campo para la arbitrariedad patronal y la manipulación política de las designaciones. (IEC-CONADU: p.11).

Pero es de observar que, avanzar hacia un proceso de normalización general que implicaría el llamado a concurso de todos los cargos actualmente interinos, podría conducir a situaciones injustas al exponer, a docentes que tras muchos años de interinato y que desempeñan adecuadamente su función, tengan que competir en un concurso abierto y perder así su trabajo. Una normalización limitada -expresa el documento (p.8)- podría permitir discriminar estas situaciones en la medida en que se determine que se llamarán a concurso los cargos más recientemente asignados y que en cambio los docentes interinos con cierta antigüedad en su cargo, accederán a la carrera a través de un mecanismo diferente que les permita regularizar su situación preservando su trabajo. Se trataría de un mecanismo de regularización "protegida" que podría ser automático o suponer un proceso de reválida.

El día $1^{\circ}$ de Agosto 2012 se comenzó a discutir con todas las representaciones sindicales del país en la Paritaria Nacional, el Capítulo del Convenio Colectivo de Trabajo correspondiente a Carrera Docente. A tal fin y con la intención de homogeneizar criterios en un proyecto único, se aprobó en el Congreso de CONADU mencionado, lo que se dio en llamar Lineamientos Mínimos para la Reglamentación de la Carrera Docente exigidos por CONADU. Allí se especifica:

- El acceso a la Carrera Docente debe efectuarse mediante el concurso público y abierto de antecedentes y oposición.

- Debe ser contemplada reglamentariamente la existencia de instancias de apelación que permitan al postulante impugnar los dictámenes de concursos y de todas las instancias de evaluación que incluya la Carrera Docente.

- Debe incorporarse en los concursos y en todas las instancias de evaluación la figura del veedor gremial.

- $\quad$ El reglamento de concursos, los mecanismos de evaluación periódica y los criterios de promoción deben establecer con claridad las exigencias correspondientes a cada categoría y dedicación, de acuerdo con el tipo de asignatura de que se trate y su modalidad de enseñanza, los objetivos de la institución, etc.

- El reglamento de la Carrera Docente debe adecuarse en todas sus instancias a la definición de funciones docentes que se estipulen en el Convenio Colectivo de Trabajo.

- No es admisible que la Carrera Docente sea opcional, o que se defina el ingreso de tal modo que haya en una institución dos clases de docentes con regímenes laborales distintos.

- Debe darse solución a la situación de los docentes interinos. Para aquellos que revistan como interinos al momento de reglamentarse la Carrera Docente, debe establecerse un mecanismo de acceso a la Carrera que 
proteja la continuidad en el cargo para quienes han desempeñado adecuadamente su función. Debe establecerse una reglamentación que limite efectivamente, en el futuro, las designaciones interinas.

- Debe definirse un mecanismo de evaluación periódica que permita acreditar el adecuado desempeño y justificar la continuidad del docente en el cargo, y que pueda además acreditar su idoneidad para ser promovido de categoría.

- Deben establecerse dispositivos intermedios de seguimiento por parte del área o cátedra a la que pertenece el docente, antes de la evaluación individual, que permitan realizar modificaciones en el desempeño o proveer los recursos necesarios para cumplir con las tareas que luego serán objeto de evaluación, y que pueden requerir apoyo institucional para poder ser llevadas a cabo.

- Es necesario que se acompañe a las trayectorias individuales con una oferta institucional de perfeccionamiento adecuada a las exigencias de la función desempeñada.

- Debe asegurarse la gratuidad de la formación de posgrado para que los docentes tengan oportunidades efectivas de perfeccionamiento en el marco de sus carreras.

- $\quad$ El régimen de promoción debe establecer en qué casos el ascenso a una determinada categoría está sujeto a concurso público y abierto de oposición y antecedentes, y cuándo la movilidad entre una categoría y la siguiente, puede producirse sin mediar concurso, sobre la base de los resultados de la evaluación periódica.

- La promoción sin concurso, que requiere haber sido evaluado satisfactoriamente en los procedimientos regulares de evaluación periódica, debe establecer claramente: qué cantidad de evaluaciones debe haber superado satisfactoriamente, y si existe alguna calificación mínima requerida, si el docente debe haber revistado cierto tiempo como mínimo en un cargo para poder aspirar a ascender, si se requiere, además, alguna evaluación presencial diferente del concurso: coloquio o entrevista, si se le pide que presente un proyecto para el desempeño del nuevo cargo.

- El cumplimiento de los requisitos definidos para ascender a una categoría superior debe generar, como mínimo, el derecho a solicitar el ascenso, sea vía concurso o a través del dispositivo de evaluación del desempeño.-

Estos "Lineamientos Mínimos" sintetizan el núcleo duro de las demandas gremiales de la etapa, en un contexto sin visos de cambio en lo que refiere al modelo universitario sostenido y respaldado por la Ley de Educación Superior (LES) vigente desde 1995. Afirmamos esto en tanto la propia diputada oficialista Dra. Adriana Puiggrós, Presidenta de la Comisión de Educación de la Cámara de Diputados, que en 2009 presentara un Proyecto de Ley Nacional de Educación 
Superior ( $N^{\circ}$ de Expediente 0458-D-2009) acompañada en ese momento por los Diputados Rossi, A.; Vaca Narvaja, P.; Damilano, V.; Leverberg, S. M.; Pasini, A.; Acosta, M.J.; Depetri, E.; Berraute, A. y Cantero, A; debió -entendemosreplegarse ante la evidencia (aún contando con mayorías "propias" en ambas cámaras legislativas) de que no habrá nueva LES en lo inmediato. Situación que la indujo presentar un nuevo Proyecto de Ley, mucho menos ambicioso en el sentido de cambio de modelo universitario, pero que valorativamente pone en otro plano el trabajo docente. Este último aborda la Regulación del ejercicio de la docencia de la Educación Superior ( $N^{\circ}$ de Expediente 3737-D2011 con trámite parlamentario 091 del 15/07/2011, acompañado por la firma de los Diputados Plaini, F.; Pilatti Vergara, M.I.; Recalde, H. y Calchaqui, M.).

Lo interesante del dato hasta donde hemos relevado la información, es que de todos los Rectores miembros del Consejo Interuniversitario Nacional, el único que ha dado respaldo abierto y público a este último Proyecto es el Dr. Marcelo Ruiz, Rector de la Universidad Nacional de Rio Cuarto. Hombre proveniente de las filas sindicales que postulan una Educación Superior Democrática, Popular, Emancipadora y Latinoamericana. Ex Secretario General de la Asociación Gremial Docente de la Universidad de Río Cuarto. Ruiz fue quien desde el 5 de Diciembre de 2007 en que se produjo la explosión de la Planta Piloto de la Facultad de Ingeniería de la UNRC, en la que fallecieron un estudiante y cinco investigadores, encabezó la más importante acción del sindicalismo docente universitario en el terreno de la defensa de las condiciones laborales llegando a constituirse esa Asociación Gremial, en querellante en la causa penal abierta. Causa en la que actualmente están imputados por estrago doloso, culposo, agravado, siete ex funcionarios de la UNRC. Actuaciones que ya han sido elevadas a Juicio, recayendo este en la Cámara Federal $\mathrm{N}^{\circ} 2$ de la ciudad de Córdoba. Aquellos hechos tuvieron que ver con investigaciones sobre el complejo oleaginoso, usando un material altamente combustible en su almacenamiento -Hexano- que proveía la Empresa De Smet. Solvente orgánico, volátil e inflamable que se encontraba albergado en forma irregular en el laboratorio.

Remarcamos estos datos porque dejan entrever claramente en qué contexto de trabajo, bajo qué amparo legal y desde qué marco valorativo, puede estarse investigando y desarrollando tareas académicas en nuestras UU.NN. Las que hacen al modelo de universidad vigente. De hecho, las discusiones mantenidas en los ámbitos gremiales indicarían que fue esta verdadera catástrofe lo que disparó en su momento la decisión del Ministerio de invertir en capacitación en Higiene y Seguridad y la repentina voluntad de los rectores -negada durante décadas- de aceptar comenzar a discutir el CCT haciéndolo por el Capítulo de Condiciones y Ambiente de Trabajo. Decisión que se tomó dejando postergada hacia adelante las cuestiones vinculadas a la estabilidad laboral. Es de destacar por otra parte, que los distintos Ministros de Educación 
que se sucedieron desde 2003 a la fecha (Daniel Filmus, Juan Carlos Tedesco, Alberto Sileoni) han asumido sistemática y públicamente, la gran deuda de una nueva Ley de Educación Superior mientras en los hechos se sigue adelante con el proceso de claro sentido privatista (en términos de "bien transable") del conocimiento público:

...proceso en que el conocimiento generado en los ámbitos públicos con financiamiento privado conlleva cláusulas de confidencialidad que limitan el libre flujo de conocimientos, así como también se estimula la selección de temas de investigación "rentables" y se valora el trabajo académico según criterios empresariales (Naidorf, J., 2009: p.24).

El esquema dominante que sigue rigiendo en las UU.NN. (y se profundiza buscando como contrapartida reducir el impacto con diversas iniciativas de "compromiso social universitario" inscripto en la perspectiva de las "políticas compensatorias") es el llamado modelo de la Triple Hélice. Este modelo propone la vinculación científico-tecnológica entre universidad y empresa, con el estado como promotor, mediador, posibilitador, facilitador o regulador de la vinculación (Llomovatte, S. 1999). El que entre otros tantos efectos, pone seriamente en cuestión el sentido unívoco con que se supo conceptualizar la noción de Autonomía Universitaria, según sea hoy la fuente de financiamiento y la demanda del mercado del conocimiento. Escenario académico institucional que presenta repercusiones inevitables en la relación de esta (la autonomía) con la evaluación. Y en consecuencia, con el valor que adquiere "lo académico" según quién y en función de qué agenda evalúa. Este es el punto que exige develar las regulaciones del proceso laboral docente en todas sus dimensiones. Entre otras cosas, las formas de producción y organización en la que se inscribe, dejando al descubierto la forma multifacética que adquiere la precarización.

\section{Reflexiones finales}

Creemos fundamental impulsar, desarrollar y multiplicar ámbitos de reflexión colectiva (no sólo a nivel mega encuentros -foros, congresos, seminarios, etc.-, sino también en pequeños grupos de investigación con más producción en red) cuya premisa en torno a revalorizar el trabajo docente, su especificidad y la enseñanza universitaria en toda su complejidad, apunten a desmontar lo que se presenta como anclado en un paradigma funcionalista adaptativo en lo que hace a la organización interna de la universidad respecto a los requerimientos del modelo de universidad comercial vigente. Es de hacer notar que ya con anterioridad a 2001 y contradictoriamente al conjunto de decisiones que se siguieron adoptando, el CIN (Consejo Interuniversitario Nacional) afirmaba que el incremento de la participación del financiamiento privado y orientado por el mercado, podía incidir, tanto en la manera de priorizar ciertas líneas de 
investigación aplicada con la intención de resolver problemas específicos de la producción, como al abandono de las investigaciones consideradas como irrelevantes o no útiles para la obtención de ganancias (7).

Sostenemos la necesidad de insistir en la promoción de investigaciones participativas con una perspectiva etnográfica, que al mismo tiempo que centren en el trabajador docente en particular desde su ubicación subjetivante, recuperen el sujeto colectivo del proceso laboral docente y sus mecanismos dadores de identidad laboral poniendo el acento en la identificación de los marcos evaluativos desde los cuales se califica y se le asigna "valor" a cada una de nuestras áreas de intervención. Esta premisa constituye una de las claves. Para lo cual, es necesario recuperar (y fortalecer) como afirmábamos más arriba, toda la dimensión intelectual de nuestra labor, con más autonomía de pensamiento, poniendo el acento en los sistemas de evaluación, sus criterios, indicadores y fundamentos políticos y epistemológicos. Es necesario de-construir el proceso mismo de alienación del trabajador docente de la ES, que, progresivamente, se ha ido asimilando (en su necesidad de "acreditar") a los mecanismos de sobreexplotación a que son sometidos el conjunto de los trabajadores -en nuestro caso- desde un nuevo y no menos contradictorio "conservadurismo" universitario que instalaron en el marco de un -cuanto menos- discutible eficientismo tecnocrático que caracteriza el modelo vigente impuesto por el llamado Estado Evaluador. Es el propio sentido de la calidad del trabajo desplegado lo que estamos observando, y cualquier generalización abstracta, puede conducirnos a consideraciones engañosas. Es de hacer notar que lo propiamente investigativo, suele estar lejos de la tarea cotidiana de la misma docencia, de sus rutinas, de las relaciones más primarias que anidan en la dinámica de la enseñanza superior.

\section{Notas Bibliográficas}

(1) Según Zúñiga Hernández en La enseñanza experta en contexto universitario. ¿Qué deja fuera en la regulación del trabajo académico (2011), el conocimiento codificado refiere a las comunicaciones escritas. Mientras que el conocimiento tácito incluye procesos intangibles como las prácticas organizacionales de un grupo de investigación o, en el caso de algunas tradiciones científicas, el montaje de laboratorios, así como el uso de determinados artefactos metodológicos.

(2) Ernest Boyer, ex presidente de la Fundación Carnegie para el Avance de la Enseñanza, en EE.UU., al señalar la diversificación del trabajo académico, plantea que la profesionalización de la investigación corresponde al trabajo académico de descubrimiento; identificando además, otros tres tipos: el trabajo de erudición (recreación del conocimiento), el trabajo de comunicación (para fines pedagógicos) y el trabajo de aplicación (solución de problemas técnicos). Según el texto mencionado (Zúñiga Hernández, Barona Ríos y otros) La diversificación de prácticas desencadenó una reflexión sobre las potenciales distorsiones del trabajo académico, incluso la incorporación de clasificaciones en la enseñanza universitaria. 
(3) Lozares, C.: Verd, J.M.; Moreno, S.; Barranco, O. y Massó, M., para reflexionar sobre los vacíos e insuficiencias de los marcos conceptuales históricamente construidos en torno a los procesos de trabajo, proponen un modelo de análisis en el que, la idea básica que subyace en torno a las aproximaciones teóricas de la Actividad Situada (AS) y del Conocimiento Socialmente Distribuido (CSD), consiste en que el conocimiento y la acción (por tanto también los procesos de trabajo) no se pueden entender ni como actividades y conocimientos puramente individuales de las personas ni como aislados de la situación en que se llevan a cabo, sino en interacción, primero, con las acciones y conocimientos de otros ( $y$ distribuidos entre) individuos y artefactos $y$, segundo, con la situación y el entorno en que se desarrollan (Conein, 1994; Suchman, 1993). Ambas aproximaciones teóricas, AS y CSD, van parejas y se complementan mutuamente en la explicación de los procesos de acción y por tanto de los de trabajo. También convergen en el sentido de que "si la cognición está distribuida, necesariamente está situada puesto que la distribución de la cognición depende en gran medida de las posibilidades situacionales" (Salomón, 1993: 114). Es en el mismo proceso de acción-reacción e interacción de los agentes al estado de la situación (y del mundo) donde se genera y distribuye el conocimiento y viceversa. Así pues, no se puede mantener desde el punto de vista pragmático, aunque sí desde el punto de vista analítico, la separación entre lo que es acción y lo que es cognición como tampoco entre lo individual y lo colectivo, ni entre la acción, la situación y el contexto estructural de su realización puesto que todo ello, en su génesis y desarrollo constituye un tejido y/o círculo complejo que mutuamente se alimenta y al mismo tiempo se abre al exterior teniendo su núcleo en la interacción.

(4) Ver "La codificación mercantil del conocimiento según las recomendaciones de los organismos internacionales" (Juarros, F.; Naidorf, J. y Guelman, A., 2006) en "Las actividades de investigación y desarrollo (I\&D) en las universidades públicas". La vinculación universidad-empresa: miradas críticas desde la universidad pública (Llomovate, S. y otros, Cap. II).

(5) Red de Estudios de Trabajo Docente. Reúne investigadores latinoamericanos del tema, desde 1999. (www.redestrado.com)

(6) Según Gómez, S. (2009) Usos y supuestos políticos teóricos de la pertinencia de la educación superior en los proyectos por una Ley de Nacional de Educación Superior (Argentina). Su inscripción en el dinámico escenario político. Tesis de Maestría. "La Pertinencia (del francés: petinence, del inglés: relevance) de la Educación Superior es introducida por los documentos especializados de la UNESCO desde 1995 y reforzada sistemáticamente a partir de 1998. Es sabido que dicha categoría cuenta con un peso creciente en la elaboración y definición de las políticas de Educación Superior. Su aparición frecuente en documentos de organismos internacionales, proyectos legislativos, etc. que refieren a la Educación Superior es una ilustración de ello. Uno de los atributos más significativos de la PES es su carácter equívoco. Al igual que otras categorías, su definición no es acabada, condensando un sin fin de significados posibles. Se presenta como un significante vacío (Naidorf, et. al. 2007). Es sustancial entender que los sentidos de las categorías que orientan políticas educativas no son casuales o contingentes sino el producto de luchas sociales generales que tienen su materialización (siempre dinámica) en ellas. De este modo, los sentidos de la PES se conforman como un objeto de disputa y se juegan en el estado de las correlaciones de fuerzas. Además es preciso reconocer que el poder de nominación de los sectores sociales es nítidamente desigual (Bourdieu, 1990). La capacidad efectiva de dar visibilidad, crear o bien de decir de cierta manera a las categorías y objetos sociales, expresa un poder político - social".

(7) Coraggio, J.L.; Vispo, A. (2001). Contribución al estudio del Sistema Universitario Argentino. Bs.As., Argentina: Miño y Dávila. 


\section{Referencias Bibliográficas}

- ADIUC-AGD-COAD. (2008). Reflexiones del Congreso Nacional por una Educación Superior Democrática, Popular, Emancipadora y Latinoamericana. Hacia la construcción de un nuevo movimiento nacional de trabajadores docentes universitarios. Córdoba: GraficArce.

- $\quad$ Antunes, R. (2005). Los Sentidos del Trabajo. Ensayo sobre la afirmación y la negación del trabajo. Buenos Aires: Herramienta Ediciones/TEL-Taller de Estudios Laborales.

- $\quad$ Bleichmar, S. (2005). La subjetividad en riesgo. Buenos Aires: Topía.

- Borón, A. (2008). Consolidando la Explotación. La academia y el Banco Mundial contra el pensamiento crítico. Córdoba: Editorial Espartaco.

- Colominas, N. (2012). La ilusión del Trabajo. Buenos Aires. Nac \& Pop. Red Nacional y Popular de Noticias. www.nacionalypopular.com/index.php?option=com

- $\quad$ Campos V.S. (2005). Flexibilización laboral de la docencia universitaria y la gest(ac) ción de la universidad sin órganos. Un análisis desde la subjetividad laboral del docente en condiciones de precariedad. En Espacio público y privatización del conocimiento. Estudios sobre políticas universitarias en América Latina. Gentili. P. y Levy, B. (comp.). Buenos Aires: CLACSO.

- Díaz Villa, M. (2006). Hacia una sociología de las competencias. Mimeo. Santiago de Cali.

- IEC-CONADU (2012). La Carrera Docente en las Universidades Argentinas. Documento aprobado por el Congreso Extraordinario de CONADU, Formosa, 22/06/2012. Documentos para el debate. Buenos Aires. IEC-CONADU.

- Imen, P. (2008). Políticas Educativas y modos de trabajo docente en Argentina: un recorrido por las imposiciones y resistencias entre la reproducción y la emancipación. Florianópolis - SC. Revista Perspectiva. Vol.26, №2.

- La Serna, C. A. (2010). La transformación del mundo del trabajo. Representaciones, prácticas e identidades. Buenos Aires: CLACSO - Ediciones CICCUS

- Llomovatte, S. y otros. (2006). La vinculación universidad-empresa: miradas críticas desde la universidad pública. Buenos Aires: Miño y Dávila Editores - Laboratorio de Políticas Públicas.

- Lozares, C.; Verd, J. M. y otros. (2004). El proceso de trabajo desde las perspectivas de la actividad situada y del conocimiento socialmente distribuido. Barcelona. Cuadernos de Relaciones Laborales. Vol.22 №1. 67-87 Grup d’Estudis Sociológics sobre la Vida Quotidiana i el Treball (QUIT). Universitat Autónoma.

- Martinez, D. (2001). Abriendo el presente de una modernidad inconclusa. Treinta años de estudios del trabajo docente. Washington DC. Latina American Studyes Association (LASA). XXIII International Congress.

- Martinez, D., Amieva, R., Gretter, S. y otros. (2009). Subjetividad y Trabajo Docente en la Universidad. San Luis. Revista Fundamentos en Humanidades. Año X NoII pp. 135/160. UNSL.

- $\quad$ Morin, E.(1998). Introducción al Pensamiento Complejo. Barcelona: Gedisa.

- $\quad$ Naidorf, J.; Martinetto, A. y otros. (2010). Reflexiones acerca del rol de los intelectuales en América Latina. Archivos Analíticos de Políticas Educativas. Vol.18, №25. http://epaa. asu.edu/ojs/article/view/730 Arizona State University.

- Naidorf, J. (2009). Los cambios en la cultura académica de la universidad pública. Buenos Aires: EUDEBA.

- Neffa, J. C. (1990). El Proceso de Trabajo y la Economía de Tiempo. Buenos Aires: Humanitas. 
- Pérez Mora, R. y Monfredini, I.; (coord.) (2011) Profesión Académica. Mecanismos de regulación, formas de organización y nuevas condiciones de producción intelectual. Universidad de Guadalajara. Jalisco, México: Ediciones de la Noche.

- Puiggros, A. y otros. (2011). Ejercicio de la docencia de la Educación Superior: Regulación: Modificación de los artículos 11 y 12 de la Ley 24521. Proyecto de Ley. H. cámara de Diputados de la Nación. Argentina. Nº de Exp. 3737-D-2011. Trámite Parlamentario $091(15 / 07 / 2011)$.

- Rama, C. (2006). La Tercera Reforma de la educación superior en América Latina. Buenos Aires: Fondo de Cultura Económica de Argentina.

- Salomon, G. (1993). Distributed Cognition: Psychological and educational considerations. Cambridge, UK: Cambridge University Press.

- Slaughter, Sh. And Leslie, L. (1977). Academic Capitalism: Politics, Policies \& the Entrepreneurial University. Baltimore: The Johns Hopkins University Press.

- Tello, A. M. (2008). Nuevas regulaciones en el trabajo docente, en ADIUC-AGD-COAD, Reflexiones del Congreso Nacional por una Educación Superior Democrática, Popular, Emancipadora y Latinoamericana. Córdoba: GraficArce.

- Torres, C. A. (Comp.) (2001). Paulo Freire e a agenda da Educaçao Latino Americana No Século XXI. Buenos Aires: CLACSO.

- Varsavsky, O. (1994). (8 ed.). Ciencia, política y cientificismo. Buenos Aires: CEAL.

- Vasilachis de Gialdino, I. (2007). Condiciones de trabajo y representaciones sociales. El discurso político, el discurso judicial y la prensa escrita a la luz del análisis sociológico-lingüístico del discurso. Argentina. Discurso y Sociedad, Vol.1, №1, 148-187. www.dissoc.org

- Zemelman, H. (2004). ¿En qué andan las ciencias sociales? Chile: Instituto de Ciencias, Artes y Literatura Alejandro Lipschütz.

- Zúñiga Hernández, O. Y.; Barona Ríos, C.; Ponce de León, O. G.; Abascal Zorrilla, M. L. (2011). "La enseñanza experta en contexto universitario. ¿Qué se deja fuera en la regulación del trabajo académico". Cap.VI. Pps.107-119. En Profesión académica. Mecanismos de regulación, formas de organización y nuevas condiciones de producción intelectual. Universidad de Guadalajara. Jalisco, México: Ediciones de la Noche. 